\title{
¿ES POSIBLE PENSAR LA MIGRACIÓN Y EL REFUGIO DESDE LA HOSPITALIDAD KANTIANA?
}

\author{
Is it possible to think the migration and the refuge \\ from the Kantian hospitality?
}

\author{
María del Rosario Guerra González* \\ Maribel Sánchez Matías*
}

\begin{abstract}
Resumen. El presente artículo polemiza sobre la categoría "hospitalidad" dentro del pensamiento kantiano, particularmente en el texto Hacia la paz perpetua (en su complementación con La Metafísica de las costumbres). En atención al problema de los refugiados y con gran visibilidad en Europa, se aprecia el conflicto entre las formulaciones teóricas de Kant -la hospitalidad como categoría moral y su desarrollo en la formulación del derecho de asilo proclamado por la Convención sobre el Estatuto de los Refugiados- frente a la legitimidad de la soberanía de los Estados, en tanto éstos deciden o no acoger refugiados.
\end{abstract}

Palabras clave: hospitalidad; refugiados; derecho de visita; apropiación originaria común del suelo; soberanía.

\begin{abstract}
The present article discusses the category of hospitality in the Kantian thought, particularly in the text Perpetual peace (in its complementation with The Metaphysics of Morals). In attention of the current problem of refugees and with great visibility in Europe, the conflict is represented between the theoretical formulations of Kant -the hospitality as moral category and its development in the formulation of the right of asylum proclaimed by the Convention Relating to the Status of Refugeesagainst the legitimacy of the sovereignty of the States, as long as they decide or not to host refugees.
\end{abstract}

Keywords: hospitality; refugees; right of visit; common original acquisition of land; sovereignty.

\footnotetext{
* Profesora-investigadora del Instituto de Estudios sobre la Universidad (IESU), de la Universidad Autónoma del Estado de México (UAEMéx). Toluca, México.

** Universidad Autónoma del Estado de México (UAEMéx). Toluca, México.
} 


\section{Introducción}

La tragedia de los migrantes y refugiados provenientes de Oriente y de África se encuentra con la respuesta de la Unión Europea mediante el cierre de sus fronteras, de eso que Nair (2016) denomina la "muralla europea", lo cual exige abordar la situación de estos desplazados bajo una nueva perspectiva: ética, pero a la vez, como un deber legal. La siguiente reflexión nace de cuestionar por qué la migración y en su especificidad, el refugio, se torna un problema. A pesar del derecho de asilo consagrado en la Declaración Universal de los Derechos Humanos y del compromiso que tienen los Estados de acoger a refugiados, estos últimos se enfrentan a problemas de acceso a este derecho en tanto lo que impera en los Estados de acogida son políticas migratorias hostiles en contraposición a las de hospitalidad. Es así que bajo este enfoque se analizará la categoría de hospitalidad desde el planteamiento kantiano, así como su vinculación y/o desarrollo en el derecho de asilo proclamado en la Declaración de los Derechos Humanos, como por la Convención del Estatuto de los Refugiados.

El texto consta de cuatro partes: En primer lugar, se hace una presentación del texto Hacia la paz perpetua; seguidamente, se analiza la categoría de hospitalidad en la obra citada; en tercer lugar, se desarrolla el planteamiento del derecho de visita en la obra La metafísica de las costumbres. Al final, se anotan las imprecisiones sobre el derecho de hospitalidad en relación con el derecho de asilo, particularmente en la violación del principio de "non refoulement" apuntado por Benhabib.

\section{Kant y el proyecto de la paz perpetua}

La referencia a la hospitalidad en la actualidad exige atender la doctrina del derecho cosmopolita kantiano. En Hacia la paz perpetua (2010b), Kant ofrece el marco teórico y conceptual que sirven de guía en la comprensión del derecho de asilo e inmigración, lo que a su vez permite delinear, en lo que atañe a este estudio, sobre las delimitaciones e imprecisiones entre el deber moral y/o legal respecto a los refugiados, en tanto el derecho de hospitalidad en la perspectiva kantiana va a situarse entre ambos.

La idea del texto es lograr la consolidación de la paz entre los Estados del mundo mediante la adecuada asociación de éstos -como aproximación a una República Universal-, consistente en abandonar la guerra de forma definitiva. Se parte de la premisa universal de que el hombre guiado por su razón práctica puede renunciar de manera voluntaria al estado de naturaleza y plantearse la paz como un fin a la vez como un deber. Para el logro de la asociación de Estados propuesta, Kant apunta a condiciones preliminares y definitivas, mismas que son bosquejadas en las dos secciones que comprehende el texto. 
Los artículos preliminares destacan las reglas o leyes para la contención de la guerra entre los pueblos, con el objetivo de establecer relaciones entre los Estados que permitan delimitar el espacio de la posible legitimación del recurso bélico, ideas necesarias, a su vez, para la consolidación de la paz perpetua ${ }^{1}$.

Cabe hacer un paréntesis para señalar las características de las guerras del presente siglo. Como apunta Pleite Guadamillas, respecto del siglo XX, lo peor de aquellas, además de los millones de muertos y refugiados que habían dejado, fue que el hombre asimiló como algo natural y consustancial a su existencia la violencia y el horror. Sin embargo, las guerras del siglo XXI han dejado de tener límites. "Ya no se diferencia entre combatientes y no combatientes, soldados y civiles, sino que toda la población se ve afectada por la guerra" (Pleite Guadamillas, 2017, p. 45). De igual manera, las guerras de este siglo, a diferencia de las precedentes, ya no consisten en invasiones de unos países por otros. Se trata, por el contrario, de guerras civiles provocadas por el debilitamiento de los Estados, así como en las divisiones entre los pueblos. Los conflictos bélicos como en Siria denotan la lucha por el control geopolítico de un territorio por distintos países. Algo además novedoso en estos conflictos es el terrorismo que azota sobre las democracias y que busca tambalear sus estructuras. Producto de esto son, precisamente, las migraciones provenientes del sur del Mediterráneo y de Asia: Siria, Afganistán, Pakistán, Eritrea, República Democrática de Congo, Nigeria. Hombres y mujeres huyen, por tanto, de las guerras y matanzas ejecutadas por grupos terroristas como ISIS o Boko Haram.

Al regresar a Kant, la segunda sección del texto abarca los "artículos definitivos", mismos que apuntan a la instauración de un orden de convivencia cosmopolita; es decir, se establece una relación de continuidad entre la política interior de un Estado y la política exterior, entre el derecho público, el derecho de gentes y el derecho cosmopolita. El primer artículo definitivo señala que todo Estado debe poseer una Constitución republicana², en tanto ésta permite a los ciudadanos obedecer las leyes a las que han brindado su consentimiento.

1. La prohibición de que existan reservas secretas en los tratados de paz; 2. La prohibición de que un Estado pueda ser adquirido por otro mediante herencia, intercambio, compra o donación; 3. La idea de la desaparición paulatina de los ejércitos; 4. La prohibición de que el Estado contraiga deuda en atención a su política exterior; 5. Todo Estado queda impedido de intervenir en la política y asuntos de otro; 6. Ningún Estado en guerra debe permitirse el uso de ciertas hostilidades que hagan imposible la reciproca confianza en la paz futura (asesinatos, envenenamientos, etc).

2 Si bien el carácter republicano es propio de la mayoría de los Estados modernos, contrario a Kant, la propuesta de Rawls es más abarcante respecto a la concepción de la organización de dichas naciones o sociedades. Al pensar en que, en el derecho de gentes, los pueblos se hallan bajo diferentes gobiernos y con cierto margen de autonomía e igualdad respecto a los otros gobiernos. El autor parte de la concepción de una sociedad liberal democrática y se extiende hacia tipos de sociedad que sin ser liberales se rigen bajo la concepción de justicia, entendida como bien común: pueblos no liberales, pero decentes (Rawls, 2002, p. 14). 
La constitución debe estar regida bajo tres principios: el principio de la libertad de los miembros de una sociedad en cuanto hombres, el de la dependencia de éstos respecto a una única legislación común, y el principio de la igualdad de todos en tanto ciudadanos. Se plantea la división de poderes con el fin de evitar el despotismo y, como consecuencia, en lo que concierne a las relaciones de orden internacional, los integrantes de cada Estado podrán decidir respecto a la legitimidad de la declaración de guerra -y su continuidad- en función de sus intereses particulares y enfatizando sobre los costos económicos de aquella, en caso de ejecutarse. El segundo artículo establece la necesidad de la existencia de una federación de pueblos que vincule a los diferentes Estados entre sí alrededor del mantenimiento de la paz. Tal liga de Estados tendrá como objetivo principal administrar el estado de paz entre todos sus miembros de manera tal que éste se establezca como una finalidad universal y no como una mera contingencia. Ello implicaría sumar progresivamente a todos los pueblos de la tierra bajo la noción de una alianza permanente para evitar la guerra $^{3}$. Finalmente, el tercer artículo, "la ley de la ciudadanía mundial debe estar limitada a condiciones de una hospitalidad universal", hace depender la factibilidad del derecho cosmopolita de las condiciones de hospitalidad universal, lo cual atañe al trato que se brinda al extranjero en un territorio que no es el suyo.

\section{La noción de hospitalidad}

Bajo estas consideraciones, interesa enfocar el trabajo en el tercer artículo definitorio, en él, Kant analiza el término hospitalidad. En primer lugar, la hospitalidad no hace referencia a una cuestión de filantropía, sino de derecho:

hospitalidad significa aquí el derecho de un extranjero a no ser tratado hostilmente por el hecho de haber llegado al territorio de otro. Este puede rechazar al extranjero, si ello no acarrea la ruina de éste, pero mientras el extranjero se comporte amistosamente en su puesto, el otro no puede combatirlo hostilmente. No hay ningún derecho de huésped en el que pueda basarse esta exigencia (para esto sería preciso un contrato especialmente generoso, por el que se le hiciera huésped por cierto tiempo) sino un derecho de visita, derecho a presentarse a la sociedad,

3 En otro texto de 1793, Kant ya señala: "Así como la general violencia, y la necesidad resultante de ella, terminaron haciendo que un pueblo decidiese someterse a la coacción que la razón misma le prescribe como medio, esto es, someterse a leyes públicas e ingresar a una constitución civil, también la necesidad resultante de las continuas guerras con que los Estados tratan una y otra vez de menguarse y sojuzgarse entre sí ha de llevarlos finalmente, incluso contra su propia voluntad, a ingresar a una constitución cosmopolita; o bien, por otra parte, si cierta situación de paz/ universal... resulta todavía más peligrosa para la libertad, por producir el más terrible despotismo, esta necesidad les llevará entonces a una situación que no es, ciertamente, la de una comunidad cosmopolita sometida a un jefe, pero sí es una situación jurídica de federación con arreglo a un derecho internacional comunitariamente pactado" (Kant, 2010a, p. 295). 
que tienen todos los hombres en virtud del derecho de propiedad en común de la superficie de la tierra, sobre la que los hombres no pueden extenderse hasta el infinito, por ser una superficie esférica, teniendo que soportarse unos junto a otros y no teniendo nadie originariamente más derecho que otro a estar en un determinado lugar de la tierra. (Kant, 2010b, p. 319$)^{4}$

Kant distingue entre el derecho de huésped y el derecho de visita. Al hacer tal distinción, el término hospitalidad corresponde al derecho de visita que se establece de manera temporal. El filósofo parte de la articulación de la posesión común de la tierra, lo que implica un derecho original de todos los hombres a la posesión de una parte de ésta. De lo anterior se sigue el derecho a intentar contacto con otros hombres y pueblos ajenos, en tanto la superficie esférica de la tierra obliga a su aproximación y contacto, en palabras de Kant, "los hombres no pueden extenderse hasta el infinito" (Kant, 1999). El derecho de visita, por tanto, implica no ser tratado con hostilidad al llegar a un territorio.

Para entender este derecho, Kant recurre a algunas premisas. La primera inscribe este derecho de visita sobre la base de la capacidad de todos los seres humanos de asociarse: "Un derecho, sin embargo, que en cuanto a la facultad de los extranjeros recién llegados, no se extiende más allá de las condiciones de posibilidad de intentar un tráfico con los antiguos habitantes" (Kant, 2010b, p. 319). La segunda y vinculada con la anterior, recurre a la construcción jurídica de la común posesión de la superficie de la tierra: "derecho a la superficie, que pertenece conjuntamente a la especie humana, para un posible tráfico" (ibidem; ver además nota 6). Más adelante se volverá a este punto.

\footnotetext{
${ }^{4}$ Respecto al término propiedad, vale la pena señalar que su referencia hace alusión a la común posesión de la tierra. Es decir, la tierra como perteneciente de manera comunitaria a todos. Porque los seres humanos están en el planeta tierra y todos, sin excepción tienen el derecho de estar en ella y visitar sus lugares y los pueblos que la habitan. Se puede establecer al respecto, la diferencia entre propiedad y usufructo. La primera consiste en el derecho real que es atribuido al titular de un bien sobre la facultad de disponer, usar y gozar del mismo dentro de los límites de la legalidad; el usufructo refiere a la disposición, uso y gozo de un bien que no se posee en propiedad, pero del cual se obliga a la conservación. El usufructo es más conveniente a la justificación kantiana del derecho de hospitalidad. Cf. Biblioteca jurídica, "propiedad", "usufructo", en Diccionario jurídico de derecho, 2014. Disponible en: <http:// www.enciclopedia-juridica.biz14.com/d/propiedad/propiedad.htm>. Consultado: 15.10.2017. Puede verse, además, la traducción de la Biblioteca Cervantes: "Significa hospitalidad el derecho de un extranjero a no recibir un trato hostil por el mero hecho de ser llegado al territorio de otro. Éste puede rechazarlo si la repulsa no ha de ser causa de la ruina del recién llegado; pero mientras el extranjero se mantenga pacífico en su puesto no será posible hostilizarle. No se trata aquí de un derecho por el cual el recién llegado pueda exigir el trato de huésped -que para ello sería preciso un convenio especial benéfico que diera al extranjero la consideración y trato de un amigo o convidado-, sino simplemente de un derecho de visitante, que a todos los hombres asiste: el derecho a presentarse en una sociedad. Fúndase este derecho en la común posesión de la superficie de la tierra [el subrayado es mío]..." (Kant, 1999).
} 
Lo que guía el tratamiento del opúsculo, y por ende, las condiciones del derecho de visita, se enfoca en la proyección de la materialización de la paz, dados los episodios bélicos de su tiempo, y, a la vez, en la expansión del comercio de forma pacífica por los diversos Estados. Empero, más allá de estos ejes, la hospitalidad planteada por Kant no obedece a la generosidad de uno hacia los de afuera de un territorio, a los extranjeros, sino como lo señala Benhabib (2015, p. 30), es

un derecho que pertenece a todos los seres humanos en la medida en que los vemos como participantes potenciales en una república mundial... este derecho regula las interacciones de individuos que pertenecen a entes civiles diferentes, pero que se encuentran el uno con el otro en los márgenes de las comunidades circunscritas.

Es un modo de cambiar la conducta hacia los extranjeros y salida del estado de naturaleza, así como la condición necesaria para lograr la paz perentoria entre los Estados y ciudadanos. La paz vista como un imperativo de derecho categórico (Loewe, 2017, p. 2018).

Bajo las consideraciones antecedentes, se anota el tránsito inminente de los individuos entre Estados diferentes, lo cual implica que un extranjero que pase por un territorio ajeno genere un derecho de hospitalidad, derecho que posibilita la convivencia cosmopolita.

Lo que está implicado en este sentido, a decir de Benhabib, y seguido por Rodríguez Ortiz, es la idea de la libertad externa (jurídica) ${ }^{5}$ que en la interpretación de Benhabib es la verdadera premisa justificadora de la doctrina kantiana en torno a la fundamentación del derecho cosmopolita (Rodríguez Ortiz, 2016, p. 140).

Dado que, sin embargo, el ejercicio de nuestra libertad externa significa que tarde o temprano, bajo ciertas circunstancias, necesitaremos cruzar fronteras y entrar en contacto con seres humanos de otras tierras y culturas, debemos reconocer lo siguiente: primero, que la superficie de la tierra será distribuida entre los territorios de repúblicas individuales, segundo, que son necesarias condiciones de derecho que regulen transacciones intraasí como interrepublicanas y, finalmente, que entre estas condiciones se encuentran aquellas correspondientes a los derechos de hospitalidad y permanencia temporaria. (Benhabib, 2015, p. 35)

De lo anterior se deduce que la negativa de entrada a un Estado deviene en la violación del derecho y sus repercusiones en el terreno de la justicia global, en tanto la obligación de hospitalidad pertenece al orden del "derecho público de la humanidad". En palabras de Kant: "como se ha avanzado tanto en

\footnotetext{
5 "Mi libertad exterior (jurídica)... [se entiende] como la facultad de no obedecer ninguna ley exterior sino en tanto en cuanto he podido darle mi consentimiento" (Kant, 2010b, nota n. 5, p. 319).
} 
el establecimiento de una comunidad (más o menos estrecha) entre los pueblos de la tierra que la violación del derecho en un punto de la tierra repercute en todos los demás" (Kant, 2010b, p. 321).

No obstante, en la configuración de dicho derecho, los Estados mantienen su soberanía y no se someten a leyes públicas (coactivas) de un orden superior. En la federación de naciones a la que apunta el filósofo no hay ninguna pretensión jurídica reclamable por parte de los Estados implicados (Habermas, 1999, p. 151). Por lo cual no se entiende cómo es posible garantizar el "derecho público de la humanidad" en la que se implica el derecho de hospitalidad sin una institución que obligue a los Estados miembros sobre leyes que permitan la protección de tales derechos. El deber de hospitalidad no puede imponerse a costa de la voluntad del soberano político de cada Estado. Es así que si se apela a este derecho de hospitalidad, ¿de qué modo respondería Kant ante el problema del flujo masivo de refugiados que huyen de Estados políticos no necesariamente republicanos, como se puede ver en las circunstancias actuales? ¿Están los Estados obligados a acoger con hospitalidad a los recién llegados? Si la respuesta es afirmativa, ¿en qué condiciones? ¿Se puede condicionar el derecho de hospitalidad apelando a la conservación de la identidad europea frente a inmigrantes y refugiados musulmanes?

Desde el enfoque kantiano, en la idea de la paz perpetua, el problema de la migración, como se plantea en la actualidad, no tendría cabida. La cuestión tendría que resolverse analizando el contexto de las sociedades de donde provienen los inmigrantes y en su solución mediante la consecución hacia Estados republicanos. Si lo que plantea el filósofo es la agrupación de las sociedades en una Federación de naciones con el objetivo de lograr un equilibrio a nivel mundial, la pretensión no se sostiene dada la relación asimétrica entre los Estados, en específico, en la injerencia de los Estados fuertes y poderosos con respecto a la salida de los inmigrantes y refugiados de los Estados débiles (explotación de recursos naturales, intervenciones humanitarias y violación de derechos), así como en el poder de cada Estado de regular sus propias fronteras ${ }^{6}$.

\section{La precisión del suelo como fundamento del derecho de visita}

Ahora bien, la fundamentación del derecho cosmopolita de hospitalidad se puede ver complementada con el texto La Metafísica de las costumbres. En la "Introducción a la doctrina del derecho", Kant menciona el derecho innato, "lo mío y tuyo interno", mismo que será relevante en la complementación de

\footnotetext{
6 Roxana Rodríguez Ortiz sostiene que el planteamiento kantiano sobre el derecho cosmopolita es insuficiente al intentar llevarlas a la práctica debido a las desigualdades económicas existentes entre los Estados que comparten una o más fronteras (Rodríguez Ortiz, 2016, p. 140-141).
} 
la construcción del principio de la posesión originaria del suelo ${ }^{7}$, que a su vez funciona como premisa en el derecho de visita desarrollado en Hacia la paz perpetua.

En el apartado la "Doctrina del derecho", Kant parte del principio de la posesión originaria del suelo para articular y limitar el derecho de propiedad. En palabras de Kant: "Todos los hombres están originariamente (es decir, antes de todo acto jurídico del arbitrio) en posesión legítima del suelo, es decir, tienen derecho a existir allí donde la naturaleza o el azar los ha colocado (al margen de su voluntad)" (Kant, 1989, p. 78). En concordancia con lo anotado en Hacia la paz perpetua, existe una posesión originaria común del suelo en tanto la tierra es limitada y los hombres no pueden extenderse al infinito, lo que lleva a la inevitable interacción de los individuos. Al seguir la interpretación de Bertomeu, esta noción de la posesión originaria del suelo es "una idea a priori, derivada del postulado de la razón práctica" (Bertomeu, 2004, p. 144).

De acuerdo con Tomassini (2015, p. 444), quien retoma este extracto:

La posesión originaria del suelo como "un derecho a existir allí donde la naturaleza o el azar los ha colocado" podría prestarse a confusión. Es importante indicar que la posibilidad de interpretar un derecho natural o innato a la posesión de tierras, a partir de este pasaje, no es plausible sencillamente porque aquí Kant no se está refiriendo a la posesión jurídica del suelo, ni a su adquisición.

El razonamiento sobre dicha posesión originaria antecede a todo acto jurídico, vinculado, por tanto, con un derecho innato: el derecho que posee el hombre de vivir u ocupar un lugar de la tierra. La filósofa argentina retoma a Kant para aludir a este tipo especial de posesión empírica: "el que quisiera quitarme de la mano la manzana en el primer caso (el de la posesión empírica) o expulsarme de mi sitio, me lesionaría en lo mío interior (la libertad), pero no en lo mío exterior..." (Kant, apud Tomassini, 2015, p. 444). Se desprende, en palabras de la autora, que "todos los hombres retienen un derecho innato e igual a 'existir allí donde el azar o la naturaleza lo ha colocado' en la medida en que ocupar un lugar en la tierra es algo propio de la existencia humana" (ibidem, p. 444-445).

Bertomeu, por su parte, conecta este derecho originario común del suelo con la libertad en la medida que el primero deriva del segundo. Dirá que "quien carece de propiedad -interna o externa-también carece de independencia, no es sui iuris" (Bertomeu, 2004, p. 143). La autora analiza la libertad y el carácter

\footnotetext{
Dentro de los estudios sobre Kant, en lo que corresponde a la filosofía del derecho, se ha enfatizado sobre el criterio normativo (jurídico) que trae la idea de la "posesión común originaria" sobre la regulación de la propiedad privada. Tomassini y Bertomeu plantean el derecho común de posesión originaria como criterio normativo de la razón práctica, mismo que hace posible un uso legítimo de los objetos exteriores al arbitrio (Bertomeu, 2004; Tomassini, 2015).
} 
de la propiedad privada de acuerdo con la Declaración de los Derechos del Hombre y del Ciudadano de 1789 para ahondar sobre la posición asumida por el filósofo prusiano respecto a la cuestión de la existencia de un derecho natural de propiedad. Concluirá, en concordancia con Kant, que "la posesión originaria del suelo [...] significa que es una consecuencia de la libertad (externa) entendida como la independencia respecto al arbitrio constrictivo de otro, en la medida que pueda coexistir con la libertad de cualquiera, según una ley universal" (ibidem, p. 144). No obstante, la independencia inferida en la libertad innata necesita el aseguramiento de "un derecho a «estar ahí» donde la naturaleza o el azar los han colocado, «un derecho a existir» que sólo puede serle garantizado mediante la posesión de una parcela del suelo común" (ibidem) derecho que pertenece a un orden cosmopolita, como principio universal de derecho. Loewe, sin albergar la garantía de la existencia en la posesión de una parcela del suelo común, la acentúa en el principio de la posesión originaria común del derecho natural sobre la base de la corporalidad humana (Loewe, 2017) ${ }^{8}$. La idea parte de la división general de los derechos que establece Kant en la "Introducción a la Doctrina del derecho". El primer orden de éstos refiere a preceptos sistemáticos (derechos objetivos), que a su vez se dividen en positivo y natural. El segundo orden de derechos (derechos subjetivos) considerados como facultades para coaccionar, se divide en innato y adquiridos. En relación al derecho innato (interno), éste se expresa en dos formas: en la posesión al propio cuerpo (sui iuris) y el honor natural de ser un hombre íntegro (iusti) (Kant, 1989, p. 48). De acuerdo a su lectura, el derecho al propio cuerpo implica "un derecho original a poder estar en algún lugar de la superficie de la tierra, y el límite del suelo obliga a un principio de derecho, de acuerdo al cual 'los hombres sólo pueden ocupar un sitio de la tierra de acuerdo a leyes de derecho'" (Loewe, 2017, p. 228). Es relevante el planteamiento de Loewe en tanto amplía el derecho de visita sobre la precisión del suelo como extensión del derecho original al propio cuerpo, lo que incluiría además, aludiendo a la interpretación de Tomassini, entender la configuración del derecho natural de tener que ocupar un lugar en la tierra como consustancial a la existencia humana ${ }^{9}$. Su no reconocimiento o violación plantearía el daño sobre la libertad en tanto el cuerpo es parte de aquella.

8 Cabe señalar que Loewe tiene un artículo antecedente, titulado "Los náufragos de nuestro mundo: el caso de los refugiados", en el que interpreta el derecho cosmopolita de hospitalidad kantiano y ahonda en el argumento de la corporalidad humana, así como en su conexión sobre la posesión originaria del suelo (Loewe, 2010).

9 En la interpretación de Tomassini, la configuración de tal derecho natural está lejos de constituir como justificación de la propiedad privada (teniendo como base la libertad como derecho innato), más bien alude a la relación constitutiva entre el suelo y la existencia (cf. Tomassini, 2015, p. 445). 
Si se piensa en los refugiados actuales, quienes, orillados a salir de sus lugares de origen para preservar su vida, llegan a las costas de Europa, éstos requieren de un derecho de visita en tanto, al igual que los náufragos a los que refiere Kant, "precisan de un suelo bajo sus pies" para seguir viviendo ${ }^{10}$. De ahí que este derecho original a la posesión del suelo repercuta en los modos de relación con los otros una vez que se rebasan las fronteras de territorios delimitados.

La reconstrucción argumentativa kantiana del derecho innato como base para un derecho humano de visita llevaría a configurarlo en términos de un derecho de hospitalidad. Sin embargo, en la construcción de dicho planteamiento se pueden vislumbrar sus limitaciones si se piensa en las políticas de ingreso en los Estados nación actuales. Por un lado, Kant coloca el derecho innato como un derecho anterior a todo ejercicio jurídico, es decir, se halla entre el orden moral y el legal. La segunda reside en que tal derecho de hospitalidad se ve limitado por las políticas de ingreso y pertenencia de los Estados naciones, que en su mayoría se ven configuradas por un criterio jurídico denominado jus sanguinis consistente en otorgar la ciudadanía -y en la repercusión sobre la facilidad de entrada y residencia- a un individuo por el hecho de la filiación sanguínea de los padres. Esta política se mantiene en países como Italia y Alemania, países de entrada y destino final de refugiados, respectivamente. De ahí la posición de Sartori respecto al mantenimiento de esta política y en el señalamiento de la inintegrabilidad de los kurdos en Alemania y del temor a que los refugiados africanos lleguen a Europa con fines de quedarse (Sartori, 2016).

\section{La idea de hospitalidad ante la crisis de los refugiados}

Como señala Alvarado Ávila, un principio kantiano es retomado en el preámbulo de la Declaración Universal de los Derechos humanos, cuando se manifiesta que la dignidad humana es intrínseca en el ser humano. En la terminología kantiana, tal dignidad es innata en los hombres y por tanto fundada en un principio a priori (Kant, 1994, p. 92). De igual manera, en el pensamiento kantiano pueden observarse conceptos clave como la libertad, derecho y lo que enfatiza Alvarado, el concepto de deber, encarnado en el término "compromiso" (Alvarado Ávila, 2006, p. 213) expresado en la Declaración Universal de los Derechos Humanos cuando "Considerando que los Estados miembros se han comprometido a asegurar, en cooperación con la Organización de las Naciones Unidas, el respeto universal y efectivo

\footnotetext{
${ }^{10}$ Loewe, en la ampliación del derecho de hospitalidad, hace uso de este argumento del derecho interno al propio cuerpo en el ejemplo siguiente: "De este modo [...] aquel que, a causa de un naufragio, es arrojado a la costa de un Estado ajeno, tiene un derecho de visita, porque precisa de un suelo bajo sus pies" (Loewe, 2017, p. 228).
} 
a los derechos y libertades fundamentales del hombre". Uno de los derechos inalienables del ser humano es precisamente el derecho de asilo. No obstante, en la práctica lo que prevalece son políticas de deshumanización no sólo de los ilegales, sino de aquellos que por su contexto se ven orillados a salir de sus países para salvar su vida. Es así como se sobrepone la soberanía de los Estados en términos de dirimir sobre sus fronteras frente al derecho fundamental de salvaguardar a los que necesitan protección.

El concepto de derecho de permanencia temporal, que correspondería al derecho de hospitalidad, será incorporado en la Convención de Ginebra sobre el estatuto de los Refugiados, específicamente en el artículo 33 de la Convención, el principio de "non refoulement", el cual establece que "ningún Estado Contratante podrá, por expulsión o devolución, poner en modo alguno a un refugiado en las fronteras de territorios donde su vida o su libertad peligre por causa de su raza, religión, nacionalidad, pertenencia a determinado grupo social o de sus opiniones políticas" (ACNUR, 1951). Dicho principio es visiblemente violado por los Estados receptores, en primer lugar, mediante la interpretación que éstos hagan de la vida y libertad adecuadas a sus intereses; segundo, en el ordenamiento de depositar a refugiados y asilados en terceros países seguros, lo que también está sujeto a una interpretación más abierta de lo que se considere un país seguro. Valga señalar como ejemplo el pacto de la deshonra entre Alemania y Turquía en marzo de 2016. Éste consistió en negociaciones entre la Unión Europea (gestionadas por Alemania) y el primer ministro turco Ahmet Davutoglu, sobre el retorno de todo "migrante" que llegase a islas griegas a partir del 20 de marzo del año anterior y su inmediata salida hacia Turquía (Nair, 2016, p. 98). Nair lo denomina pacto deshonroso dado que se hablaba de retorno de refugiados que el acuerdo los transformó en inmigrantes. La ley que imperó no fue la de las convenciones sobre refugiados sino las migratorias europeas (Schengen, Dublin, etc).

Este acuerdo plantea que todos los nuevos migrantes irregulares que pasen de Turquía a las islas griegas a partir del 20 de marzo de 2016 serán devueltos a Turquía; las autoridades griegas tramitarán las solicitudes de asilo individualmente, en cooperación con el ACNUR. Los migrantes que no soliciten asilo o cuya solicitud se haya considerado infundada serán retornados a Turquía; por cada sirio retornado a Turquía de las islas griegas, se reasentará a otro sirio procedente de Turquía en la UE; toda necesidad adicional de reasentamiento, más allá de las 18.000 plazas existentes, se abordará a través de un acuerdo voluntario similar para alcanzar un límite máximo de 54.000 personas más. (Los países de la UE deberán dar su acuerdo de reasentamiento sin estar obligados a reasentar). (ibidem, p. 99-100) 
Es decir, la prioridad de la Unión Europea ha sido la vigilancia y el control de los migrantes y refugiados, no así la protección y cuidado de éstos. En ello, también el Frontex ha tenido un papel relevante.

Otro ejemplo que sirve para evidenciar el escenario de violación de este artículo se da con el acuerdo entre la Unión Cristianodemócrata (CDU) al que pertenece la canciller de Alemania Angela Merkel y el presidente de la Unión Socialcristiana (CSU), Horst Seehofer, sobre políticas migratorias y de asilo, cuyo objetivo ha sido limitar la entrada de refugiados al país, el número de acogidos por año según el acuerdo asciende a 200.000 personas. Dicho acuerdo surge en razón de las denuncias de su propio partido sobre la decisión de Merkel respecto a una política generosa entre los dos años anteriores y que ha repercutido de manera considerable en las elecciones para su reelección, particularmente, en la perdida de electores y sobre el futuro de las elecciones del siguiente año en Baviera. El acuerdo también incluye la ampliación de la lista de países considerados seguros (Marruecos, Argelia y Túnez) a donde puedan ser devueltos los solicitantes de asilo (Carbajosa, 2017). Medidas que pretenden hacer patente que no todo el que llegue a territorio europeo podrá quedarse.

Se presenta el conflicto entre las formulaciones de deber moral ante los asilados y refugiados frente a la soberanía legítima de quienes deciden o no acoger refugiados. A decir de Benhabib, las formulaciones kantianas no previeron el equilibrio sobre las dos posiciones (la necesidad e intereses). Sólo en aquellos casos donde la vida se pone en peligro si se niega el derecho al ingreso, las proposiciones son imprecisas, en otros términos, fuera de tales casos "la obligación de respetar la libertad y bienestar del visitante puede permitir una interpretación estrecha por parte del soberano a quien se dirige, y puede no considerarse un deber incondicional" (Benhabib, 2015, p. 36).

Para recapitular, los Estados que forman parte del sistema de Derecho internacional y que a su base tiene la Convención de Ginebra de 1951 y el Protocolo de 1967, tienen la obligación de ejecutar la protección primera de asilo, la cual consiste en el principio de non refoulement, a quien necesita refugio. La garantía de tal derecho se ha visto erosionada por políticas nacionalistas - proteccionistas- y xenófobas, poniendo en riesgo la integridad de millones de seres humanos que se ven orillados a dejar sus hogares a causa de los conflictos bélicos y la violencia.

¿Están los Estados obligados a acoger a los recién llegados?, ¿es permisible negar el asilo a grandes cantidades de personas necesitadas de protección en los Estados de acogida cuando su vida depende de estar en un suelo seguro para seguir viviendo? Son cuestiones que exigen atenderse en la actualidad. Kant ha hecho un aporte clave en la conceptualización de la hospitalidad: ha 
definido a ésta en el reconocimiento universal del otro, cuya relevancia tanto filosófica como práctica es patente, empero, dicha categoría como mandato universal debe reformularse al presente para atender los problemas de la migración y el refugio en su relación con las desigualdades socio-económicas y políticas entre los diversos Estados, desigualdades que se visibilizan en el fortalecimiento de las fronteras geopolíticas.

\section{Bibliografía}

ALVARADO ÁVILA, Víctor. Ética y filosofía del derecho en Kant y su influencia en la Declaración Universal de los Derechos humanos. Revista de Ciencias Jurídicas, n. 110, p. 213, mayo-agosto 2006.

BENHABIB, Seyla. Los derechos de los otros. Extranjeros, residentes y ciudadanos. Barcelona: Gedisa, 2015.

BERTOMEU, María Julia. De la apropiación privada a la adquisición común originaria del suelo. Un cambio metodológico (menor) con consecuencias políticas revolucionarias. Revista Isegoría, n. 30, p. 127-134, 2004. Disponible en: $<$ http://isegoria.revistas.csic.es/index.php/isegoria/article/viewFile/479/479>.

CARBAJOSA, Ana. Merkel pacta un objetivo máximo de 200.000 refugiados al año. El País, Internacional, Berlín, 09.10.2017. Disponible en: <https://elpais.com/ internacional/2017/10/09/actualidad/1507540614_541377.html>.

KANT, Immanuel. La metafísica de las costumbres. Traducción y notas de Adela Cortina y Jesús Conill Sancho. Madrid: Tecnos, 1989.

KANT, Immanuel. Fundamentación de la metafísica de las costumbres. Madrid: Espasa Calpe, 1994.

KANT, Immanuel. La paz perpetua. Traducción de F. Rivera Pastor. Alicante: Biblioteca Virtual Miguel de Cervantes, 1999. Disponible en: <http://www. cervantesvirtual.com/nd/ark:/59851/bmchd7r6>. Consultado: 11.10.2017.

KANT, Immanuel. En torno al tópico: eso vale para la teoría, pero no sirve de nada en la práctica. Traducción Roberto R. Aramayo. Madrid: Gredos, 2010a.

KANT, Immanuel. Hacia la paz perpetua. Traducción de Jacobo Muñoz. Madrid: Gredos, 2010b. Versión utilizada para las citas del texto.

LOEWE, Daniel. Los náufragos de nuestro mundo: el caso de los refugiados. ARBOR Ciencia, Pensamiento y Cultura, Santiago de Chile, p. 555-570, julio-agosto 2010. Disponible en: < https://doi.org/10.3989/arbor.2010.744n1217>.

LOEWE, Daniel. Libertad y propiedad en la fundamentación del Estado y el cosmopolitismo kantiano. In: ORMEÑO KARZULOVIC, Juan; VATTER, Miguel (eds.). Forzados a ser libres. Kant y la teoría republicana del derecho. Chile: Fondo de Cultura Económica, 2017, p. 209-231.

HABERMAS, Jürgen. La inclusión del otro. Estudios de teoría política. Barcelona: Paidós, 1999. 
NAIR, Sami. Refugiados. Frente a la catástrofe humanitaria, una solución real. Barcelona: Crítica, 2016.

PLEITE GUADAMILLAS, Francisco. Europa. Entre el miedo y la hospitalidad. Cantabria: Sal Terrae, 2017.

RAWLS, John. El derecho de gentes. España: Paidós, 2002.

RODRÍGUEZ ORTIZ, Roxana. Los límites de la hospitalidad en las fronteras geopolíticas contemporáneas. In: ARTEAGA CONDE, Evelia; OLIVA RÍOS, Mariela; RODRÍGUEZ ORTIZ, Roxana (compiladoras). Hospitalidad y ciudadanía. De Platón a Benhabib. México: Ítaca, 2016, p. 140-141.

SARTORI, Giovanni. La carrera hacia ningún lugar. México: Taurus, 2016.

TOMASSINI, Fiorella. El concepto de 'posesión común originaria' en la doctrina kantiana de la propiedad. Anales del Seminario de Historia de la Filosofía, v. 32, n. 2, p. 435-449, 2015. Disponible en: <https://revistas.ucm.es/index.php/ ASHF/article/viewFile/49972/46449>.

\section{Documentos Jurídicos}

Alto Comisionado de las Naciones Unidas para los Refugiados (ACNUR). Convención sobre el Estatuto de los refugiados de 1951. Disponible en: < http://www.acnur. org/fileadmin/scripts/doc.php?file=fileadmin/Documentos/BDL/2001/0005>.

Alto Comisionado de las Naciones Unidas para los Refugiados (ACNUR). Protocolo sobre el Estatuto de los Refugiados. ACNUR. Disponible en: < http:// www.acnur.org/t3/fileadmin/scripts/doc.php?file=t3/fileadmin/Documentos/ BDL/2001/0003>.

Recibido para publicación en 15.04.2018

Aceptado para publicación en 02.08.2018

Received for publication in April 15 $5^{\text {th }}, 2018$

Accepted for publication in August 02 ${ }^{\text {nd }}, 2018$

ISSN impresso 1980-8585

ISSN eletrônico 2237-9843

http://dx.doi.org/10.1590/1980-85852503880005313 